\title{
Femtosecond fiber CPA system in a single pass configuration
}

\author{
Michał Laskownicki, ${ }^{1}$ Jan Szczepanek, ${ }^{1}$ Yuriy Stepanenko, ${ }^{1,2}$ Piotr Skibiński, ${ }^{1}$ Piotr Wasylczyk ${ }^{2}$ Michał Nejbauer, ${ }^{* 1}$ \\ and Czesław Radzewicz ${ }^{1,2}$ \\ ${ }^{1}$ Institute of Physical Chemistry, Polish Academy of Sciences, Kasprzaka 44/52, 01-224 Warszawa, \\ ${ }^{2}$ Institute of Experimental Physics, Faculty of Physics, University of Warsaw, Hoża 69, 00-681 Warszawa
}

Received September 03, 2013; accepted February 24, 2014; published March 31, 2014

\begin{abstract}
We present a compact Yb-doped fiber chirped pulse amplification system set in a single pass configuration. The system is capable of delivering $34 \mu \mathrm{J}$ pulses with $200 \mathrm{kHz}$ repetition rate. When seeded with a low average power oscillator, the amplifier optical-tooptical efficiency is as high as $37 \%$ and the gain reaches $52 \mathrm{~dB}$ Temporal pulse degradation due to self-phase modulation is explored One stage amplification allowed us to limit the set-up complexity, typical of multi-stage CPA systems
\end{abstract}

Fiber lasers and fiber amplifiers are growing in importance as they can provide both high average output power and good beam quality at the same time [1]. On the other hand, single mode fiber geometry imposes fundamental limits on pulse energy due to limited extractable energy in the fiber, nonlinear pulse distortions and damage issues. In the case of femtosecond pulse amplification fiber nonlinearities are the primary limiting factor because of long propagation paths and small beam diameters.

The chirped pulse amplification (CPA) concept is commonly used to overcome this detrimental effect [12]. Recently, several modifications have been introduced - pulse shaping [3] or misaligned stretcher and compressor configuration [4] - to push pulse energy limits even further. Most of the setups reported so far utilize two or more amplification stages [5-7] to obtain tens of $\mu \mathrm{J}$ energy levels with a reduced $(<1 \mathrm{MHz})$ repetition rate. Other works explore the limits of the highest possible pulse energy or average power $[3,8]$ at the expense of significant complication of the amplifier set-ups.

Here we describe a simplified system where femtosecond pulses from a low average power modelocked oscillator are amplified in a single pass configuration in a $\mathrm{Yb}$-doped single mode large mode area (LMA) fiber, reaching $50 \mu \mathrm{J}$ at $200 \mathrm{kHz}$. To reduce nonlinear effects, the CPA technique is used, but with moderate-size optical elements and thus a modest pulse stretching ratio. We show how the temporal characteristics of the output pulse is affected by fiber nonlinearities.

The system consists of a passively mode locked femtosecond oscillator, a grating stretcher-compressor

*E-mail: nejbauer@ichf.edu.pl pair, an electro-optical modulator (Pockels cell) pulse picker and an active medium - single mode $\mathrm{Yb}$-doped photonic crystal fiber set in a single pass configuration.

The femtosecond oscillator is presented schematically in Fig. 1. The original design is described in [9]. The active medium of the oscillator is 1.2-mm Brewster-cut $\mathrm{Yb}: \mathrm{KYW}$ crystal pumped with a 980nm single mode fiber coupled laser diode emitting $560 \mathrm{~mW}$ of average power. Passive mode-locking is obtained by introducing a semiconductor saturable absorber mirror SESAM [10] in the place of a cavity end mirror. The resonator generates pulses of $350 \mathrm{fs}$ duration at $94 \mathrm{MHz}$ repetition rate. The central wavelength is set to $1030 \mathrm{~nm}$ with a $4.3 \mathrm{~nm}$ FWHM bandwidth. The average output power is about $90 \mathrm{~mW}$ and the beam quality factor is $\mathrm{M}^{2}<1.1$.

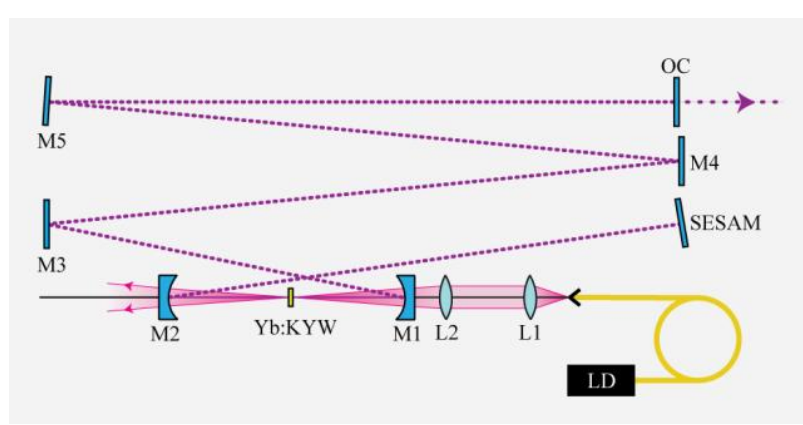

Fig. 1. Seeding Yb:KYW femtosecond oscillator, where: LD - laser diode; L1, L2 - lenses; M1, M2 - dielectric concave mirrors (ROC = $100 \mathrm{~mm})$; M3-M5 - chirped flat mirrors; OC - 5\% output coupler.

The schematic of the CPA amplifier is presented in Fig. 2. The seeding pulses are directed into the grating stretcher in the Martinez geometry [11]. The stretcher is "folded" by the mirror M3 and uses the concave mirror M2 with a 500mm focal length. The diffraction grating with 1700 grooves $/ \mathrm{mm}$ is $52 \mathrm{~mm}$ wide. Pulses are stretched to about 170ps FWHM. The mirror M4 is tilted so that the beam enters and leaves the setup through the same mirror M1.

The train of chirped pulses passes the BBO electrooptical modulator where the repetition rate is reduced to 200kHz. The Pockels cell works in a double-pass configuration with a quarter-wave voltage (about $2 \mathrm{kV}$ ) 
applied to the BBO crystal for about 10ns. Two drivers working in the tandem-mode allow us to pick the pulses with high contrast $(>30 \mathrm{~dB})$.

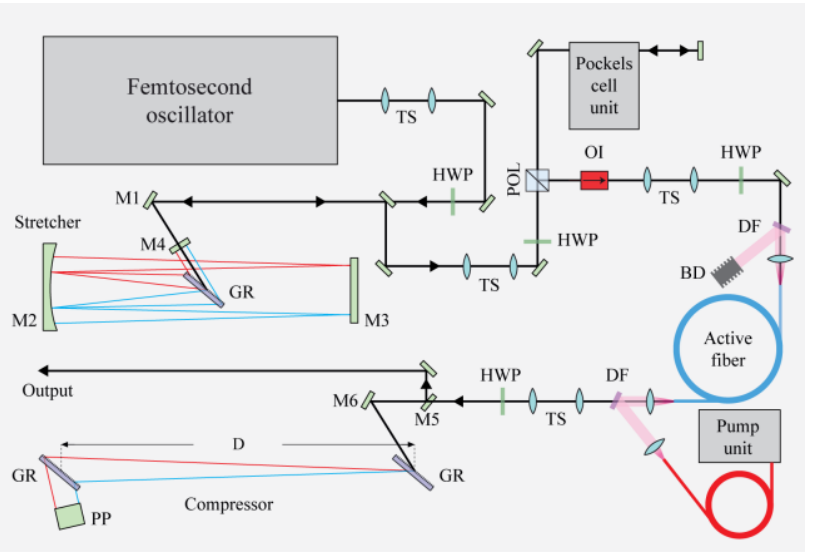

Fig. 2. Chirped pulse amplification system, where: TS - telescope; HWP - half-wave plate; GR - diffraction grating; POL - polarizer; OI optical isolator; DF - dichroic filter; $\mathrm{BD}$ - beam dump; PP - Porro prism.

The active medium of the amplifier is a $40 \mu \mathrm{m}$ core diameter, polarization-maintaining, single-mode, double clad ytterbium-doped photonic crystal fiber (aeroGAINFLEX from NKT Photonics). The fiber is $1.5 \mathrm{~m}$ long and terminated with high power SMA-905 connectors with copper collars for better heat dissipation at high pump powers. The signal and the pump $(976 \mathrm{~nm}$, delivered to the system with a $400 \mu \mathrm{m}$ core diameter, 0.22 NA fiber) are coupled into the active fiber from opposite ends. For the seed coupling and collimation $18 \mathrm{~mm}$ and $20 \mathrm{~mm}$ focal length aspherical lenses are used, respectively.

At the initial repetition rate of $94 \mathrm{MHz}$, about $30 \mathrm{~mW}$ of the seed reaches the amplifying stage - around one-third of the oscillator initial average power. Most of the loss is due to the limited stretcher efficiency $(60 \%)$ and the pulse picker optics. At the $200 \mathrm{kHz}$ repetition rate only about $60 \mu \mathrm{W}$ of the seed is coupled into the fiber. The amplified signal reaches $10 \mathrm{~W}$ before compression at the highest available pump power of $27 \mathrm{~W}$. This corresponds to an optical-to-optical efficiency of $37 \%$ and $52 \mathrm{~dB}$ gain in the single pass amplification. At higher pump levels the formation of nanosecond giant pulses originated from ASE is observed, disturbing the amplification process, creating spectral distortions and increasing the risk of fiber end-caps damage. Even below 27W of pump power the ASE requires careful management. We observed that the returning mirror placed in the back of the Pockels cell unit (when it is in "off" state) reflects ASE generated in the fiber core and counter propagates to the signal and directs it back into the fiber, where it undergoes further amplification. Hence an optical isolator is inserted so as to increase the minimum pump power level at which significant ASE is observed.

The final stage of the CPA system is a grating compressor consisting of two volume phase transmission gratings (the same as used in the stretcher) and a prism retroreflector (Porro prism). Its overall efficiency is $70 \%$.

Figure 3 presents the pulse output energy after compression as a function of pump power. The highest value obtained with the CPA system reaches $34 \mu \mathrm{J}$. The temporal contrast of the pulse was found to be better than $40 \mathrm{~dB}$ as measured using an ultrafast photodiode and a set of calibrated filters.

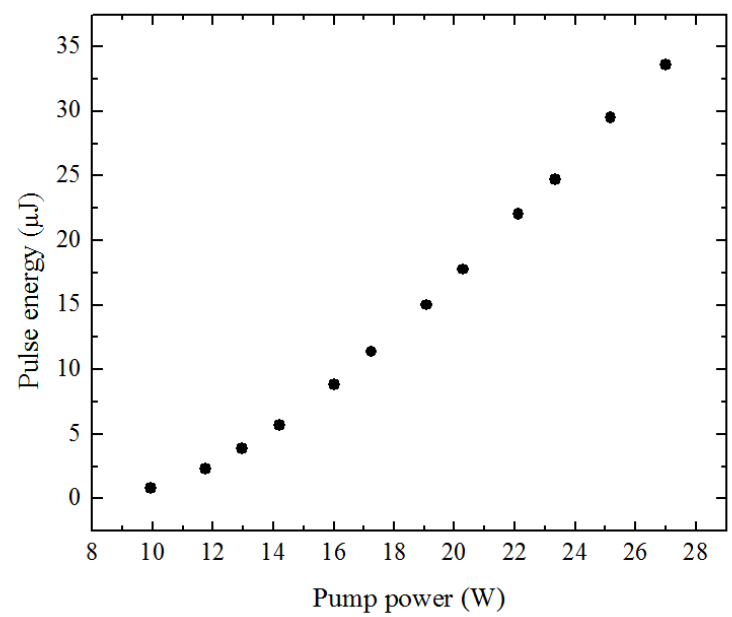

Fig. 3. Overall CPA pulse output energy vs. pump power measured at the repetition rate of $200 \mathrm{kHz}$.

The initial distance $\mathrm{D}$ between the gratings in the compressor is set for low energy levels. Above ca. $5 \mu \mathrm{J}$ of the compressed pulse energy, a new distance is found that gives shorter pulses. The optimum distance was found by observing the second harmonic signal. At each energy point the autocorrelation traces were measured (Fig. 4). Since the temporal profiles for higher pulse energies became significantly distorted, the FWHM measure is not a good measure of the pulse duration. Therefore, to quantify the autocorrelation traces widths, we adopted the RMS definition from [12]:

$$
\tau_{r m s} \equiv \sqrt{\left\langle t-\langle t\rangle^{2}\right\rangle}=\sqrt{\left\langle t^{2}\right\rangle-\langle t\rangle^{2}}
$$

where:

$$
\left\langle t^{n}\right\rangle \equiv \int_{-\infty}^{\infty} t^{n} I(t) d t
$$

We note that for the Gaussian autocorrelation profile the frequently used FWHM value corresponds to $2.35 \tau_{\text {rms }}$. 
A few examples of measured autocorrelation traces are shown in Fig. 4. Based on autocorrelation width, the duration of the compressed pulses is 450fs FWHM at $2 \mu \mathrm{J}$ and increases to $700 \mathrm{fs}$ at $8 \mu \mathrm{J}$. Even at the highest energy, where the temporal profiles are highly distorted, the output pulse spectra do not exhibit any visible distortion (Fig. 5). The results of the dispersion tuning are shown in Fig. 6. For the highest pulse energies the improvement in pulse duration is more than $50 \%$. The power dependent optimum distance between the compressor gratings indicates that the induced second order nonlinear dispersion is compensated, leaving third and higher orders uncompensated.

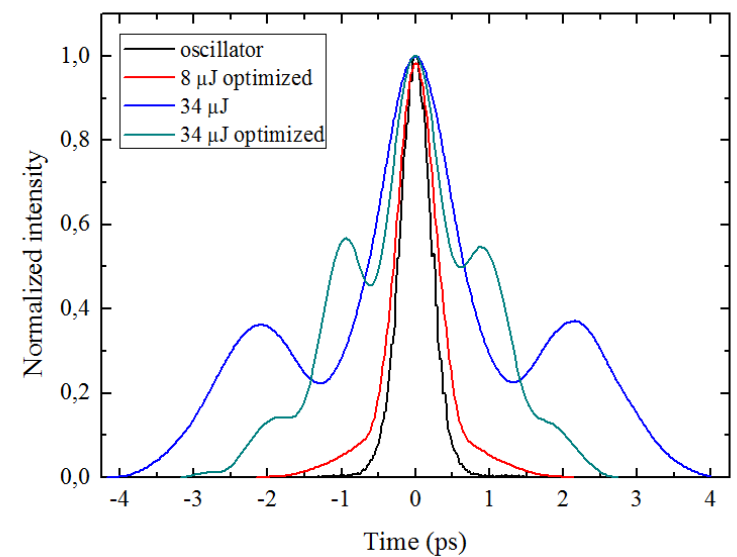

Fig. 4. The seeding oscillator and the CPA output pulse autocorrelations measured for different pulse energy. See text for details.

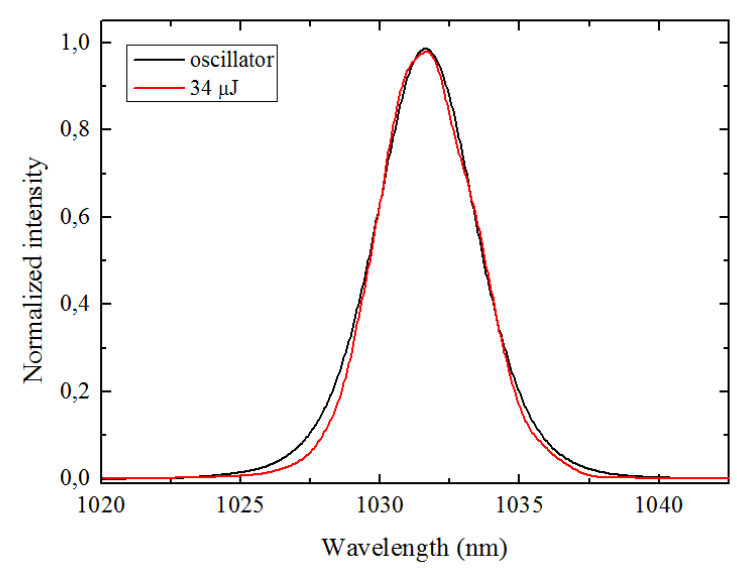

Fig. 5. Measured seeding oscillator and the high power CPA pulse spectra.

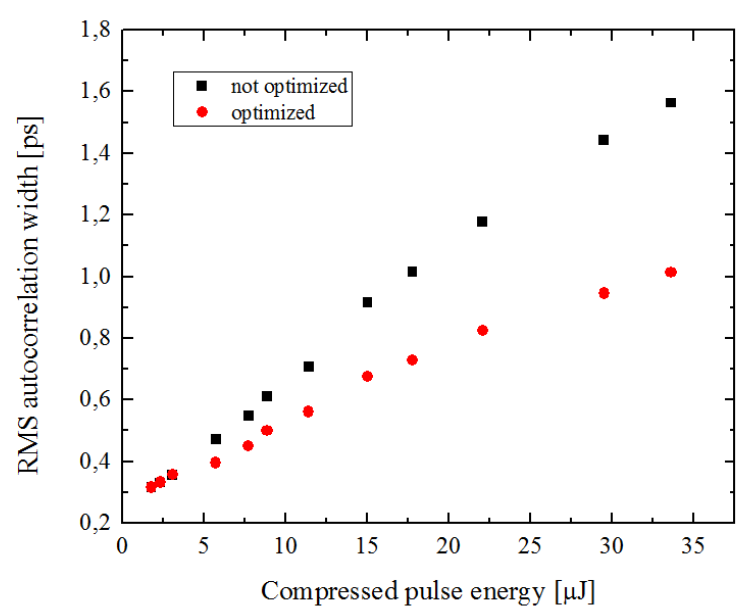

Fig. 6. The CPA output pulse duration before and after compressor optimization by adjusting the distance between compressor gratings.

We have presented the design and performance of a femtosecond $\mathrm{Yb}$ :fiber CPA system simplified to a single stage (single pass) configuration. The stretcher and the compressor units are constructed from inexpensive, medium-sized optical elements. The amplifier is seeded with a low average power $\mathrm{Yb}: \mathrm{KYW}$ oscillator resulting in high gain amplification preserving high temporal contrast. As expected, nonlinear effects strongly affect the temporal profile of the compressed pulses at high energies, but this can be further reduced by stretching the pulses more or by compensating higher orders of nonlinear dispersion. The presented femtosecond system can be used in a variety of applications, ranging from nonlinear laser spectroscopy to femtosecond pulse material processing.

The research was co-financed by the European Regional Development Fund under the Operational Programme Innovative Economy (POIG.01.03.01-22080/09).

\section{References}

[1] J. Limpert et al., IEEE JSTQE 13, 537 (2007).

[2] D. Strickland, G. Mourou, Opt. Commun. 56, 219 (1985).

[3] A. Klenke et al., Opt. Lett. 38, 2283 (2013).

[4] Y. Zaouter, J. Boullet, E. Mottay, E. Cormier, Opt. Lett. 33, 1527 (2008).

[5] L. Kuznetsova, F. Wise, Opt. Lett. 32, 2671 (2007).

[6] J. Želudevičius, R. Danilevičius, K. Viskontas, N. Rusteika, K. Regelskis, Opt. Expr. 21, 5338 (2013).

[7] T. Schreiber, C. Nielsen, B. Ortac, J. Limpert, A. Tünnermann, Opt. Lett. 31, 574 (2006).

[8] T. Eidam et al., Opt. Lett. 35, 94 (2010).

[9] P. Wasylczyk, C. Radzewicz, Laser Physics 19, 1 (2009).

[10] A. Jasik et al., J. Appl. Phys 106, 053101 (2009).

[11] O.E. Martinez, IEEE J. Quantum Electron. 23, 59 (1987).

[12] R. Trebino, Frequency-Resolved Optical Gating: The Measurement of Ultrashort Laser Pulses (Boston, Springer 2000). 